\title{
Experimental Research on the Effect of Ginsenoside Rb1 in Curing Rats with Spinal Cord Ischemia-reperfusion Injury Bo-yin $\mathrm{ZHANG}^{1}$, Dong-xu $\mathrm{ZHAO}^{1,{ }^{*}}$ and Chun-yang MENG ${ }^{1}$ \\ ${ }^{1}$ China-Japan Union Hospital of Jilin University, 126 Xian-Tai Street, Chang Chun 130033,China. \\ ${ }^{*}$ Corresponding author
}

Keywords: Spinal cord ischemia-reperfusion injury, Ginsenoside Rb1, Nerve injury

\begin{abstract}
Spinal cord ischemia-reperfusion injury (SCII), which was proposed by Allen in 1911, is the secondary damage of primary spinal cord injury and the main reason resulting in nerve injury complication and sequela[1]. Ginsenoside is one of the ingredients of Ginseng and the monomer composition being purified at present reached over 50 types[2-3]. The existing research proves that Ginsenoside Rb1 can play a significant role in neuro protection by alleviating oxygen radical generated from brain injury and lowering calcium influx. In this way, cerebral ischemia-reperfusion injury can be alleviated, but few researches are found in terms of spinal cord ischemia-reperfusion injury[4-6].
\end{abstract}

\section{Introduction}

Ginsenoside Rb1 is a potential therapeutic agent for the treatment of spinal cord ischemia reperfusion injury (SCII), although it has not yet been investigated in depth. The aim of the present study was to investigate the effects of ginsenoside Rb1 treatment on SCII and aquaporin 4 (AQP4) expression in the rat spinal cord. Experimental animals were subjected to one of four conditions, including the blank control condition, sham procedure, spinal cord ischemia reperfusion induced by abdominal aortic occlusion or spinal cord ischemia reperfusion followed by ginsenoside Rb1 treatment. Locomotor activity was evaluated using the Basso Beattie Bresnahan scale. Spinal cord damage was assessed with hematoxylin and eosin and Nissl staining and the apoptotic rate was measured using terminal deoxynucleotidyl transferase dUTP nick end labeling. AQP4 expression was assessed by immunohistochemical analysis, western blotting and reverse transcription quantitative polymerase chain reaction. Abdominal aortic occlusion resulted in the reduced expression of AQP4 in the spinal cord, which gradually recovered over time. Furthermore, ginsenoside Rbltreatment significantly attenuated this decrease and protected the integrity of and reduced the apoptotic rate in spinal cord neurons. Treatment withginsenoside $\mathrm{Rb} 1$ attenuated the initial downregulation and advanced the recovery of AQP4 expression levels, suggesting a possible mechanism for the therapeutic effects on SCII.

\section{Material and Method}

\section{Experime ntal Animal and Grouping}

40 sprague-dawley rats, which belonged to clean grade, weight: $220 \pm 20 \mathrm{~g}$, half male and half female, and were provided by Animal Experimental Center of Jilin University (License: SCXK (J) 2008-0005), were randomly divided into blank control group, sham operation group, ischemia-reperfusion injury group, Ginsenoside Rb1 
treatment group. There were 10 rats in each group.

\section{Experime ntal Drug}

Ginsenoside Rb1 (purity 98.6\%) was provided by Agricultural College of Jilin University. $0.9 \%$ normal saline was applied to prepare $5 \mathrm{mg} / \mathrm{ml}$ solution for the experiment.

\section{Establishment of Animal Model}

Conduct anesthesia on rats with $10 \%$ chloral hydrate and $3 \mathrm{ml} / \mathrm{kg}$ intraperitoneal injection. Fix the rat on one side, and disinfect certain position with $75 \%$ alcohol. Conduct $3 \mathrm{~cm}$ incision on the middle line of lower left rib of rat, find out the position of left kidney, confirm the renal artery, search for aorta abdominal along the renal artery, separate aorta abdominals below the renal artery branches, ligature it with $10 \mathrm{~g}$ standard bulldog clamp for $60 \mathrm{~min}$. After $60 \mathrm{~min}$, remove the bulldog clamp, splash penicillin powder $(2 \times 105)$ IU on certain position, close abdominal cavity.

\section{Drug-delivery Way}

For Ginsenoside Rb1 treatment group, provide Ginsenoside Rb1 intraperitoneal injection everyday according to $10 \mathrm{mg} / \mathrm{kg}$ dosage. The dosage is the same everyday in 7 days. The first injection was conducted after 30 min of successful modeling.

\section{Sample Collection}

After 7 days of reperfusion, remove L3-6 segment of spinal cord in a rapid manner under general anesthesia. Each segment was divided into two parts. One was fixed by $40 \mathrm{~g} / \mathrm{L}$ formalin for $48 \mathrm{~h}$; another one was stored in cryogenic refrigerator $(-70)$ immediately for testing the content of MDA, SOD, T-AOC in tissue.

\section{Testing Index}

Neurological Scoring: After 7 days of reperfusion, hind limb neurological scoring and record were conducted on spinal cord ischemia reperfusion injury model with Basso Beatie Bresnahan(BBB) fugl-meyer assessment.

Functional Disability Score of Tilting: Plate Scoring was made by referring to the tilting plate method when Rivlin measured the motor function of rat after spinal cord injury. To eliminate the interference of anesthesia and operation as well as to improve the reliability of the result, functional score obstacle rate was adopted to evaluate the changes of motor function of animal's hind limbs. Rivlin tilting functional disability rate $(\%)=($ score before anesthesia- score after SCI) / score before anesthesia $\times 100 \%$.

\section{SOD, MDA and T-AOC Measure ment of Spinal Cord Tissue of Rats}

Select spinal cord that is stored in cryogenic refrigerator, cut about $0.2 \mathrm{~g}$. After weighting, add brine ice and homogenate according to the mass ratio of 1:9 between tissue and water. Ultraviolet spectrophotometry was adopted to measure the changes of SOD, MDA and T-AOC activity in spinal cord. The kits were product of Nanjing Jiancheng Bioengineering Institute, and the measure was conducted by completely following the measuring steps mentioned in the kit specification.

\section{Pathology Detection}

Take out formalin-fixed spinal cord tissue for paraffin embedding, slicing and $\mathrm{HE}$ staining. Observe morphological changes under the light microscope. 


\section{Statistical Method}

Data analysis and processing were conducted with SPSS17.0 statistical software and were shown in the form of (mean value \pm standard deviation). One-way analysis of variance was applied on sample mean comparison of multi-group, while LSD method was adopted on pairwise comparison of means in any two groups. If $p<0.05$, it is of statistical significance.

\section{Results}

\section{Fugl-meyer Assessment of Rats with Spinal Cord Injury}

The BBB scoring result of rat's hind limbs of each group showed that the BBB score of rat's hind limbs in blank group and sham operation group was 21; no paralysis occurred. Different levels of paralysis appeared in the rat's hind limbs in model group and Ginsenoside Rb1 treatment group. The BBB score of model group was significantly lower than the normal control group $(p<0.05)$; the BBB score of Ginsenoside $\mathrm{Rb} 1$ treatment group also decreased respectively, but was apparently better than the model group ( $p<0.05)$. (See Tab.1)

Table.1 BBB Motor Function Scores Condition of Rat's Hind Limbs in Each Group

\begin{tabular}{cc}
\hline Group & Scores \\
\hline Blank Group & $21.0 \pm 0.00$ \\
Sham Operation Group & $21.0 \pm 0.00$ \\
Model Group & $14.6 \pm 0.97^{*}$ \\
Treatment Group & $19.1 \pm 1.20^{* \#}$
\end{tabular}

$* \mathrm{p}<0.05$, compared with blank group and sham operation group; $\# \mathrm{p}<0.05$, compared with model group

\section{Functional Disability Rate of Tilting Plate}

The functional disability rate of tilting plate of rats in each group showed that the functional disability rate of tilting plate of model group was significantly higher than other groups $(p<0.05)$; the functional disability rate of tilting plate of treatment group was significantly higher than the model group, but the difference was still of statistical significance compared with blank group and sham operation group $(p<0.05)$. (See Tab. 2)

Table.2 Functional disability rate of rat's tilting plate in each group

\begin{tabular}{cc}
\hline Group & Functional Disability Rate of Tilting Plate \\
\hline Blank Group & $2.2 \pm 0.69$ \\
Sham Operation Group & $2.1 \pm 0.89$ \\
Model Group & $55.5 \pm 1.85^{*}$ \\
Treatment Group & $34.8 \pm 1.44^{* \#}$ \\
\hline , in comparison to blank group and sham operation group; \# $\mathrm{p}<0.05$, in comparison to
\end{tabular}




\section{Measure ment of SOD, MDA, and T-AOC in Rat's Spinal Cord Tissue}

Compared with blank group and sham operation group, the difference of SOD, MDA and T-AOC in model group and treatment group is of statistical significance $(p<0.05)$. In terms of MDA content in rat's spinal cord tissue, model group is significantly higher than other groups $(p<0.05)$; for the SOD and T-AOC content, the model group is lower than other groups $(p<0.05)$; for the SOD and T-AOC content, the treatment group was significantly lower than the blank group and sham operation group $(p<0.05)$.

Table.3 SOD, MDA and T-AOC Content in Rat's Spinal Cord Tissue

\begin{tabular}{llll}
\hline Group & MDA $(\mu \mathrm{mol} / \mathrm{g})$ & SOD $(\mathrm{nu} / \mathrm{mg})$ & T-AOC $(\mathrm{u} / \mathrm{mgprot})$ \\
\hline Blank Group & $4.7 \pm 0.97$ & $80.0 \pm 3.12$ & $0.146 \pm 0.026$ \\
Sham Operation Group & $4.7 \pm 0.81$ & $80.1 \pm 3.08$ & $0.140 \pm 0.029$ \\
Model Group & $14.7 \pm 1.49^{*}$ & $39.5 \pm 3.54^{*}$ & $0.033 \pm 0.009^{*}$ \\
Treatment Group & $7.8 \pm 0.83^{* *}$ & $63.6 \pm 3.37^{* *}$ & $0.066 \pm 0.008^{* *}$ \\
\hline
\end{tabular}

\section{Pathological Observation}

Observe the changes of rat's spinal cord tissue and cells under optical microscope. It showed that no changes were found in the cellular morphology of blank group and sham operation group. In model group, some nerve cells have been damaged. Some of the nerve cell soma enlarged with unclear structure, while other cell has karyopyknosis, and hyperemia was found in tissues. The pathologic changes of Ginsenoside Rb1 treatment group was improved significantly, and the cellular morphology were at normal condition. (Fig.1)
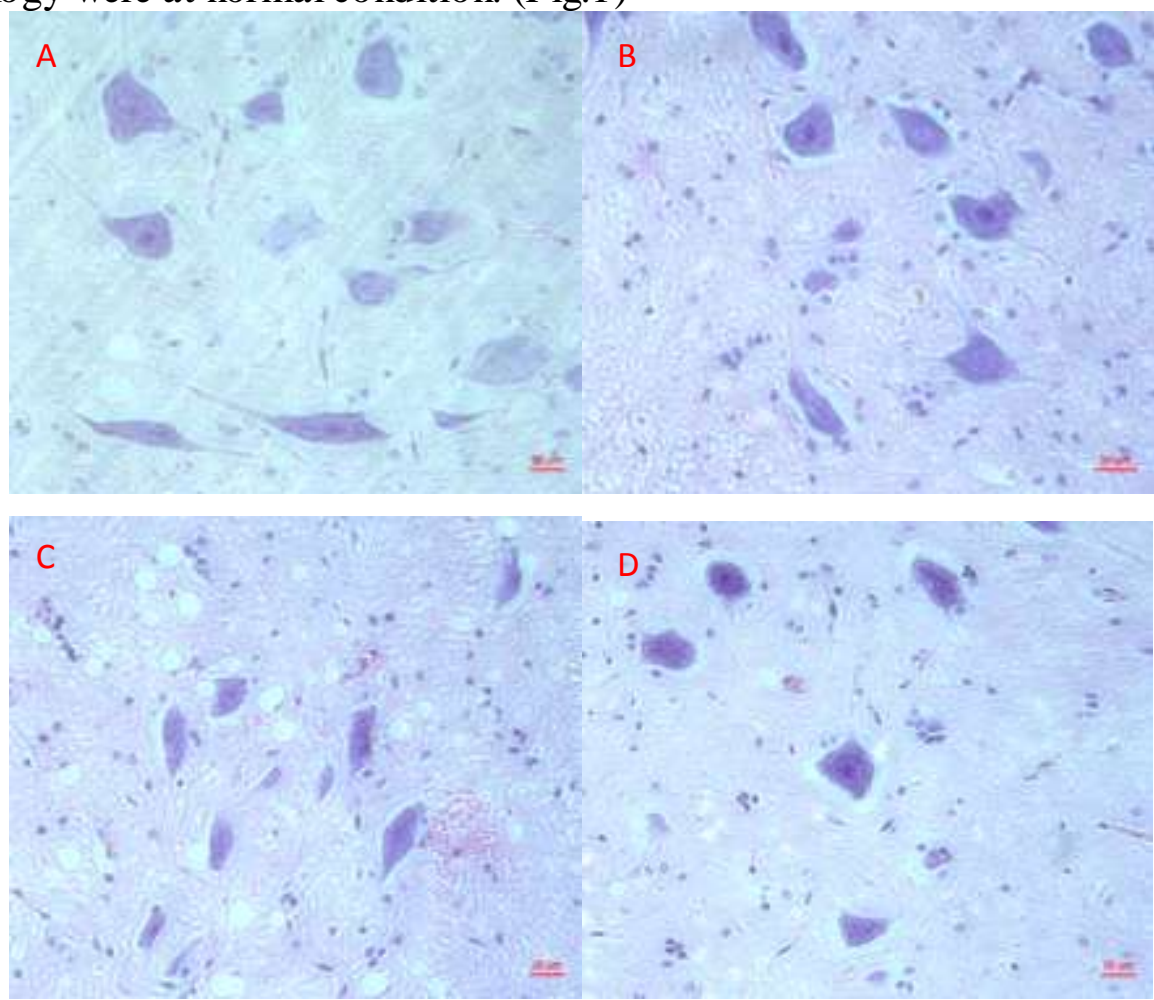

Fig. 1 Rat's Spinal Cord Tissue HE Staining (A Blank Group; B Sham Operation Group; C Model Group; D Treatment Group) 


\section{Discussion}

Spinal cord ischemia-reperfusion injury is an extremely complicated pathological process [8]. Specific molecule and cell mechanism is not clear yet, but is considered that they are related to the release of tissue excitatory amino acid[9], generation of free radical[10], overload of intracellular $\mathrm{Ca}^{2+}[11], \mathrm{Na}^{+}-\mathrm{K}^{+}$-ATP balance disorder, inflammatory reaction, nerve cell apoptosis, etc. Each factor exists at the same time and interacts with each other, resulting in injury of tissue cell. Oxidative stress reaction in spinal cord ischemia-reperfusion injury is the major reason leading to second ary injury and necrosis. Oxidative stress reaction boosts the production of free radicals, while free radicals may lead to lipid peroxidation in cell membrane. The nerve cell of spinal cord tissue, which is made up of biological membrane, is very sensitive to free radical and lipid peroxidation product[12]. These membrane structures are of vital importance to spinal cord metabolism and various physiological functions. Once cell membrane and its structure is damaged, it will lead to tissue hypoxia nerve cell degeneration, necrosis, and hemorrhagic edema, aggravating spinal cord injury[13].

Ginseng belongs to Araliaceae perennial herb and has a history of thousands years when serving as medicine. Ginsenoside Rb1 is the ingredient of ginseng, belonging to Ginsenoside of double alcohol group. Research showed that Rb1 is featured by antioxidant stress, anti-infection, anti-tumor, immunity boosting and anti-fatigue[14-15]. According to the research, Rb1 can protect heart, lung, brain, and renal ischemia-reperfusion injury[16-19].

It can be observed from the pathological slices and rat's neurological scoring result, Ginsenoside Rb1 can promote spinal cord injury recovery and neural functional recovery on rats with spinal cord ischemia-reperfusion injury.

The measuring result of SOD, MDA and T-AOC in rat's spinal cord tissue showed that Ginsenoside Rb1 can effectively improve the activity of organism SOD, lower MDA content, enhance T-AOC, and significantly reduce the formation of oxygen radical in injury tissue. Its mechanism is probably as follows: (1) Ginsenoside Rb1 directly participates in the reaction which eliminates oxygen radical in vivo. When $\mathrm{Rb} 1$ enters into the body for hydrolysis or enzymolysis, it can generate matters that can carry out redox reaction with radical ion, and directly react with free radical. As a result, it lowers free radical accumulation[20]. (2) The fact that Ginsenoside Rb1 can improve SOD enzyme activity is probably that the substrate concentration in SOD enzyme catalysis free radical redox reaction affects enzymic catalytic reaction rate, while $\mathrm{Rb} 1$ can directly participate in eliminating oxygen free radical, reducing substrate concentration of redox reaction and stimulating SOD enzymatic activity[21].

\section{Summary}

Above all, the application of Ginsenoside $\mathrm{Rb} 1$ is an effective means of clinical intervention to cure spinal cord ischemia reperfusion injury, and has promising aspect of application.

\section{Reference}

[1] Liu Yu, Zhang Jihao, Zhang Li, Conclusion on the Experience of Curing Spinal Cord Injury with Professor Zhang Anzhen[J], Traditional Chinese Medical Orthopedics Medicine, 2014, 22 (2): 63-65. 
[2] Wang HY, Qi LW, Wang CZ, et al. Bioactivity enhancement of herbal supplements by intestinal microbiota focusing on ginseno- sides[J]. Am J Chin Med, 2011, 39(6): 1103-1115.

[3] Perry E, Howes MJ. Medicinal plants and dementia therapy: herbal hopes for brain aging?[J].CNS Neurosci Ther, 2011, 17(6): 683-698.

[4] Yuan QL, Yang CX, Xu P, et al. Neuroprotective effects of ginsenoside Rb1 on transient cerebral ischemia in rats[J].Brain Res, 2007, 1167:1-12.

[5] Ji YC, Kim YB, Park SW, et al. Neuroprotective effect of ginseng total saponins in experimental traumatic brain injury[J]. J Korean Med Sci, 2005, 20(2): 291-296.

[6] Yoshikawa T, Akiyoshi Y, Susumu T, et al. Ginsenoside Rb1 reduces neurodegeneration in the peri-infarct area of a thromboembolic stroke model in non-human primates[J].J Pharmacol Sci, 2008, 107(1): 32-40.

[7] Rivlin AS, TatorCH. Objective clinical assessmentformotorfunction after experimental spinal cord injury in rat[J]. J Neurosurg, 1977, 47(5): 577-581.

[8] Gong Shouping, Application of MHP36 Neural Stem Cell Rebuilding Nerve And Blood Vessel Unit in Spinal Cord Ischemia Reperfusion [J]. Western Medicine, 2014, 26(6): 677-679.

[9] Xue Yuxian, Gao Jianfeng, Liu Ke. Influence of Electricity Needle on Nuclear Transcription Factor NF- $\kappa \mathrm{B}$ and Amino Acid Transmitters of Aging Rat's Ischemia Reperfusion Brain Tissue [J]. Traditional Chinese Medical Orthopedics Medicine, 2010, 25(3): 462-465.

[10] Tang Qun, Wu Hua, Lei Jiushi, Antioxidative and Protective Effect of Chinese Yam Polysaccharide Pretreatment on Rat's Renal Ischemia Reperfusion Injury [J]. Chinese Medical Herald, 2013, 10(9): 21-22.

[11] Ma Yuxia, Wang Shu, Ni Guangxia, Shi Xuemin, Influence of Acupuncture on Focal Cerebral Ischemia of Different Times/the Hippocampal Neuron [Ca2+] of Brain Slice in Reperfusion Injury [J]. Journal of Traditional Chinese Medicine, 2007, 35(5): 9-11.

[12] Watson F, Gasmi L, Edwards SW. Stimulation of intracellular Ca2+ levels in human neutrophils by soluble immune complexes. Functional activation of FcgammaRIIIb during priming[J]. J Biol Chem. 1997, 272(29): 17944-17951.

[13] Li Qin, Research on the Protective Effect of Ginsenoside Rb1 on Rat's Acute Spinal Cord Injury [J]. Economics of Chinese Medicine, 2013,(z1):74-75.

[14]Wang J, Qiao L, Li S, Yang G. Protective effect of ginsenoside Rb1 against lung injury induced by intestinal ischemia-reperfusion in rats [J]. Molecules, 2013, 18(1):1214-1226

[15] Zhang Juntiam, Comparison on the Pharmacological Effect of Ginsenoside Rg1 and Rb1 [J]. Preclinical Medicine and Clinical Application, 2000,20(5):388-390.

[16] Liu Zhengxiang, Liu Xiaochun, Influence of Ginsenoside Rb1 and Re on Rats with Ischemia Reperfusion Myocardial Apoptosis [J]. Chinese Histochemistry and Cytochemistry Medicine, 2002,11(4):374-377. 
[17] Di Qing, Wu Da, Zhang Bengu, et al, Protective Effect of Ginsenoside Rb1 on Ex-vivo Ischemia Reperfusion Injury and Its Mechanism [J]. Journal of Shanxi Medical University, 2005,36(3):310-312.

[18] Tang Ning, Wen Zhu, Zhang Jin et al. Research on Ginsenoside Rb1, Astragalus Membranaceus Anti-Rat Cerebral Cortical Neurons Hypoxic Apoptosis [J]. Journal of Jiangxi Med ical College, 2006,46(4):24-27.

[19] Deng Yao, Xie Xisheng, Fan Junming, et al, Antioxidative Effect of Ginsenoside Rb1 on Rat's Renal with Half Ureteral Obstruction [J]. Medical Journal of West China, 2009,21(2):196-199.

[20] Xu Junwei, Zhou Yan, Wang Dongli, et al. Experimental Research of Ginsenoside Rb1 on Anti-Motility Skeletal Muscle Fatigue [J]. Journal of Liaoning College, 2010,31(2):111-113,125.

[21]Arnold RS1, Shi J, Murad E, et al. Hydrogen peroxide mediates the cell growth and transformation caused by the mitogenic oxidase Nox1[J]. Proc Natl Acad Sci USA. 2001, 98(10): 5550-5555. 